\title{
Integrated surveillance: Joint modeling of rodent and human tularemia cases in Finland
}

\author{
Chawarat Rotejanaprasert* \\ National Center for Genetic Engineering and Biotechnology, Bangkok, Thailand
}

\section{Objective}

We seek to integrate multiple streams of geo-coded information with the aim to improve public health surveillance accuracy and efficiency. Specifically for vector-borne diseases, knowledge of spatial and temporal patterns of vector distribution can help early prediction of human incidence. To this end, we develop joint modeling approaches to evaluate the contribution of vector or reservoir information on early prediction of human cases. A case study of spatiotemporal modeling of tularemia human incidence and rodent population data from Finnish health care districts during the period $1995-2013$ is provided. Results suggest that spatial and temporal information of rodent abundance is useful in predicting human cases.

\section{Introduction}

An increasing number of geo-coded information streams are available with possible use in disease surveillance applications. In this setting, multivariate modeling of health and non-health data allows assessment of concurrent patterns among data streams and conditioning on one another. Therefore it is appropriate to consider the analysis of their spatial distributions together. Specifically for vector-borne diseases, knowledge of spatial and temporal patterns of vector distribution could inform incidence in humans. Tularemia is an infectious disease endemic in North America and parts of Europe. In Finland tularemia is typically mosquito-transmitted with rodents serving as a host; however a country-wide understanding of the relationship between rodents and the disease in humans is still lacking. We propose a methodology to help understand the association between human tularemia incidence and rodent population levels.

\section{Methods}

Data on rodent population levels are collected around the country by the Finnish Natural Resources Institute. Human Tularaemia cases are recorded as laboratory-confirmed and reported to the National Infectious Disease Register (NIDR). Human cases and rodent data were aggregated to match the 20 Finnish health districts over the period 1995-2013 [1]. We develop our methodology in a Bayesian setting. The counts of human cases for each health district in a given year are assumed to follow a Poisson distribution and the rodent data are assumed to have a categorical likelihood. The linear predictors linked to the human and rodent likelihood functions are then decomposed additively into spatial, temporal, and space-time interaction random effects. We then link the two likelihoods via the interaction term by assuming that the human spatiotemporal variation is dependent on the rodent activity with one-year lag. In the case of the rodent data, we also included two additional spatial and non-spatial contextual terms to better model ecological effects associated with rodent population levels as described before [2]. We then finally develop indicators, on the scale 0 to 1 , to quantify the association between human incidence and a rodent vector.

\section{Results}

Results suggest that spatial and temporal information of rodent abundance is useful in predicting human cases.

\section{Conclusions}

Future modeling directions are recommended to include environmental and epidemiological factors. To the best of our knowledge, this is the first time that rodent data, captured for nonhealth related purposes, is used to better inform the human risk of tularemia in Finland.

\section{Keywords}

Tularemia; surveillance; multivariate; joint modeling

\section{References}

[1] Rossow, H., Ollgren, J., Hytönen, J., Rissanen, H., Huitu, O., Henttonen, H. \& Vapalahti, O. (2015). Incidence and seroprevalence of tularaemia in Finland, 1995 to 2013: regional epidemics with cyclic pattern. Euro surveillance: bulletin Européen sur les maladies transmissibles = European communicable disease bulletin, 20(33).

[2] Sane, J., Ollgren, J., Makary, P., Vapalahti, O., Kuusi, M. and Lyytikainen, O. (2016) 'Regional differences in long-term cycles and seasonality of Puumala virus infections, Finland, 1995-2014', Epidemiology and Infection, pp. 1-6. doi: 10.1017/ S0950268816000765.

\section{*Chawarat Rotejanaprasert}

E-mail: baantoto@gmail.com 